\title{
The Net Generation: Tech-savvy or lost in virtual space?
}

\author{
Barbara Combes \\ Lecturer \\ Edith Cowan University \\ Australia
}

\begin{abstract}
Recent findings from PEW Internet and American Life studies in the US, the JISC Information Behaviour of the Researcher of the Future report and the Educational Testing Service 2006 ICT literacy assessment, all indicate that the students from the $Y$ or Net Generation are not as tech-savvy as portrayed by the world's media and large Internet software providers. If this is the case, then assumptions currently being made about the information-seeking behaviour of today's students need to be rectified at the school level to ensure that tomorrow's citizens are not disenfranchised or disempowered as users in a world where Governments are increasingly committed to the provision of essential services and information wholly online. This paper discusses the secondary results of a much larger PhD study on the informationseeking behaviour of the Net Generation and the need for schools and particularly teacher librarians, to become more involved in teaching students how to use the electronic environment effectively.
\end{abstract}

Net Generation, tech-savvy, Generation Y, digital natives

\section{Introduction}

Supporters of the Net Generation theory (also labelled the Y Generation) claim that children born after 1985 have an in-depth grasp and almost 'intuitive' knowledge of how to use technology, simply because they have never known a world without the Internet and technological change. This theory contradicts traditional information theory which contends that information-seeking behaviour is a complex activity that is affected by cultural, educational and social contexts (Case, 2002). Anecdotal evidence from schools and public libraries has long suggested that while young people actively use technology, they do not use it as described by the Net Generation theorists. In recent years there has been an emerging body of research on the Net Generation (Banwell, \& Gannon-Leary, 2000; Barr, et. al, 2006; Combes, 2006 \& 2007a; ETS, 2006; Fallows, 2005; Livingstone, et. al., 2005; Nicholas et. al. 2008) that largely debunks the myth of an intuitive user who is capable of using electronic resources to find information, a fact many teacher librarians have long suspected.

This research presents the secondary findings from a much larger $\mathrm{PhD}$ study which examines the information-seeking behaviour of young adults who fall into the upper age group of the Net Generation. The study seeks to discover whether the culture of use that surrounds the Internet has affected the way these young people use and obtain information from online and electronic resources. This paper will report on the findings from an anonymous Web survey and a series of in-depth interviews with a group of volunteer students from the Net Generation. It will discuss the information-seeking behaviour of these young adults, the skills required to use the Internet and electronic resources effectively, and how the results of this study may affect the teaching of information skills in schools. 


\section{Background}

The term Net Generation refers to children born after 1985 and was first used to describe this generation by Donald Tapscott in 1998, a time when use of the Internet was just emerging as a global phenomenon (Tapscott, 1998). Members of the Net generation are also referred to as Millennials, Generation Y or digital natives (Skiba, 2003). The underlying premise of the Net Generation theory is familiarity. The theory posits that these children and young adults are not afraid of technology and already have the skills required to use information communications technologies (ICTs) and electronic resources to seek information, simply because the technology is an everyday part of their information landscape (Tapscott, 1998; Oblinger \& Oblinger, 2005). These are the 'digital natives' of the twenty-first century whose use of technology to find information appears to be vastly different from their parents and older generations.

According to the theory, members of the Net Generation have a range of attributes that sets them apart from previous generations. Their increased access to information via the Internet and electronic resources gives this generation a greater knowledge base which fosters independence and the ability to question and confront information (Tapscott, 1998). As a result of being exposed to a lot of knowledge on the Internet this group are more socially active, responsible and discerning users of information, and they are preoccupied with free expression and have strong views (Tapscott, 1998). Members of the Net Generation know what they want and have greater digital literacy skills (Skiba, 2003; Oblinger \& Oblinger, 2005); are intuitive visual communicators, who have strong visual-spatial skills and are readily able to integrate the virtual with the physical world (Oblinger \& Oblinger, 2005). They are exploratory learners and therefore develop skills which enables them to retain information and use it in innovative ways (Skiba, 2003; Dorman, 2000; Oblinger \& Oblinger, 2005). Multi-tasking, a craving for connectivity and social engagement, the ability and a propensity to use a wide range of technologies allows the Net Generation to communicate with a broad range of users and exposes them to a wide range of ideas and cultural differences, thus leading to a more socially inclusive outlook (Tapscott, 1998; Dorman, 2000).

While there is no doubt that technology has affected and continues to affect the way we live and influences nearly every aspect of our daily lives, this body of popular literature requires closer analysis to determine whether the characteristics assigned to the Net Generation are based in fact, or are merely observations that describe what young people appear to be doing when using ICTs, rather than their actual skill levels and achievements. The basic premise of the Net Generation theory, that familiarity with technology equates with efficient and effective use and these achievements are only applicable to a specific group because they have grown up with technology, is flawed. Does this mean that children born into an era where cars are the norm, will therefore not only drive, but be good drivers simply because they have never known a landscape that is different? The theory also ignores the changing nature of technology, which has in turn produced an information landscape that is increasingly complex and populated by old and new information forms and technologies that require multiple skills to interrogate successfully.

The significance of the Net Generation literature cannot be understated. Much of it is freely available on the Internet where the nature of the medium ensures that the theory is recirculated and kept in the forefront of the popular media. It has also been used by more serious researchers and educationalists who are seeking innovative ways to cater for a generation of students who expect more from education than the traditional chalk and 
talk/lecture and content-based mode of curriculum delivery. The popular media present this generation as super users of technology and assign labels such as tech-savvy, web-savvy, Internet-savvy and computer-savvy. These terms have appeared in major educational policy documents such as the US National Technology Plan Toward a New Golden Age in American Education (U.S. Department of Education/Office of Educational Technology, 2004) and Voices \& Views from Today's Tech-Savvy Students, part of a national report sponsored by the non-profit group NetDay (NetDay, 2004; Murray, 2004). In these systemic documents today's students are assumed to have a level of proficiency when seeking and using information found on the Internet and via electronic resources. The Australian Curriculum Corporation's report from the Le@rning Federation also describes the current generation of students as capable users who are able to acquire, communicate and manipulate information, and respond creatively to new technologies (Curriculum Corporation, 2005). To continue the driving analogy, does increased access to technology mean that everyone in this group 'drive', like 'driving' and 'drive' well on the Internet? And is this really that important anyway?

Governments and information agencies worldwide are currently employing digital initiatives which will ultimately make all government information and services transparent and publicly available via egovernment Web portals. Digital information repositories are also a feature of the corporate world, as the almost ubiquitous use of technology in the workplace produces an ever increasing amount of information. The endorsement of environmentallyfriendly solutions to information storage, also make digital solutions very attractive. Supporters of egovernment solutions argue that digital information is more cost effective, easier to store (takes up less space), easier and faster to produce, easy to manipulate and with search engine technology, easier to locate. Governments also argue that information accessed by electronic means is catering for the next generation who, according the Net Generation theory, prefer and already have the skills to access information in this format. For citizens in the twenty-first century, government and public information is increasingly being published only in digital format. Being able to locate, interpret and use this information is going to be an essential skill set for citizens in the future. Thus, assumptions currently being made about the information-seeking behaviour of today's students need to be rectified at the school level to ensure that tomorrow's citizens are not disenfranchised or disempowered as users in a world where Government, education and economic information, and the provision of essential services is provided wholly online.

\section{Method}

The initial data collection consisted of an anonymous Web-based survey of first year university students between the ages of eighteen and twenty-one (upper age group of the Net Generation cohort). This empirical data provided information about:

- $\quad$ the types of software and hardware used by young people;

- $\quad$ how they use technology for study and recreational purposes;

- whether age or experience are determining factors;

- how important information communications technologies (ICTs) are in their daily lives;

- how they feel about their skill levels (self-perception and self-esteem) and where/how they acquired these skills; and

- what they principally use technology for - information gathering, communication, entertainment, organisational tool. 
The findings from this dataset were reported at the Cyberspace, D-world, E-learning: Giving libraries and schools the cutting edge, The 2007 IASL Conference, National Taiwan Normal University, Taipei, Taiwan (Combes, 2007a) and the ASLAXX Biennial Conference: Hearts on fire, sharing the passion, Adelaide, Australia (Combes, 2007b) in 2007. The initial Web survey included 533 participants, with 232 or $43 \%$ of the total survey group volunteering to be part of the follow-up study.

The initial dataset obtained from the Web survey was used to develop two metrics to determine the confidence levels (Affective Domain) and technology use (Effective Domain) of participants. It was used to target participants for a follow-up study. Contrary to the Net Generation theory, it was posited that in a normal population, the participants should fall into four categories according to their index of 'Net Gen-ness':

1. LC/LU-NG: Low Confidence, Low Use Net Gen Attributes

2. LC/HU-NG: Low Confidence, High Use Net Gen Attributes

3. HC/LU-NG: High Confidence, Low Use Net Gen Attributes

4. HC/HU-NG: High Confidence, High Use Net Gen Attributes

The Web survey was followed by a series of semi-structured, in-depth interviews and two information tasks conducted with forty students who exhibited a range of Net Gen attributes as determined by the metric. According to the Net Generation theory, all or most of the participants should have emerged in category 4: HC/HU-NG: High Confidence, High Use, Net Gen Attributes. A cluster analysis was conducted on the Web survey dataset which revealed no significant clusters for this particular group of users. Hence, a major finding from the Web survey is the fact that this group of Net Geners is very homogeneous. Rather than being very confident, high-end users of technology, they appear to be discerning and average users of technology when level of use is determined by technology type, length of use and frequency of use. Almost twenty percent of the survey group do not like using technology for learning and while males are more confident than females, the difference in confidence is not statistically significant, an indication that girls are fast closing the gender gap reported in earlier studies. When asked how they acquired their skills, most participants $(>87 \%)$ indicated they had learnt to use the Internet by experiential learning by themselves. A significant percentage of students also reported they had difficulties with simple information literacy skills such as collecting (30.206\%), managing $(28.705 \%)$ and evaluating information $(25.202 \%)$; finding information again for later use $(22 \%)$; and even storing information $(16.885 \%)$ for later use (Combes, 2007b). Almost all of the participants in the survey group fell within one standard deviation of the means for confidence (Affective Domain) and use of technology (Effective Domain) as determined by the metric. The homogenous nature of the group is obvious when the data is presented as a scattergram in Figure 1. 


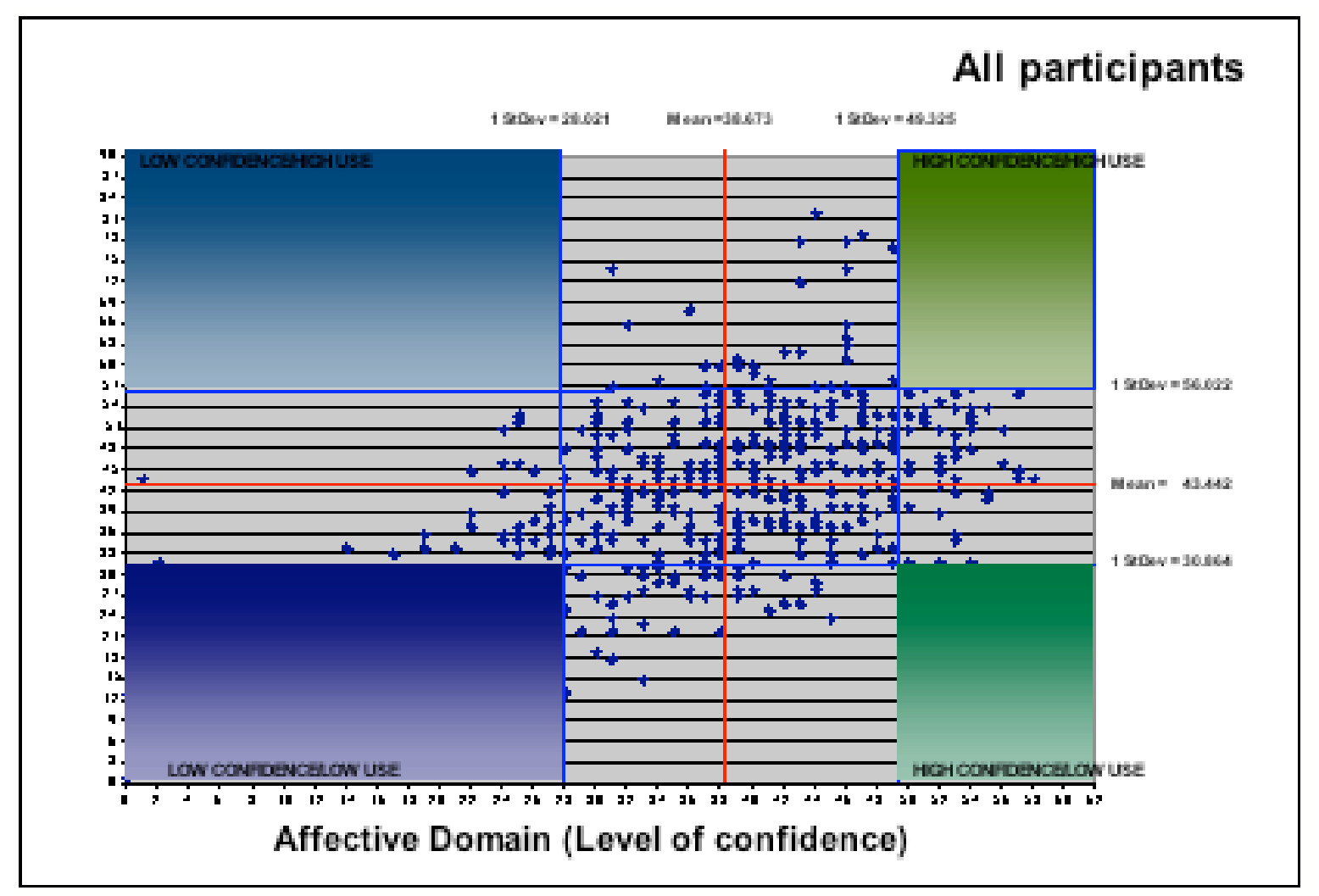

Figure 1: Net Generation attributes for all participants

\section{Follow-up tasks and interviews}

A sample of 40 students who were identified as having certain Net Gen attributes, were selected for interview. Participants were asked to complete two tasks. One task had a personal or recreational information-seeking focus (finding information for a holiday trip), while the other had an educational information-seeking focus (finding information for an essay and tutorial presentation). Hence, both tasks were set in a real life context. Verbal protocols (think alouds) were used to track the participants' information-seeking behaviour and thought processes. Use of the software program Morae, allowed the researcher to photograph the participant, record verbal protocols and track their information-seeking on the computer. The software allowed the tasks to be conducted in a less intrusive manner and enabled the researcher to compare participants' body language and dialogue as they verbalise their actions (what they think they're doing) with their information seeking behaviour real time (as it is happening) by tracking and recording their use of the technology.

Wherever possible, the task analysis was followed by semi-structured, in-depth interviews. The interviewer used a structured interview format and an interview prompt checklist to provide consistency and to act as a quality assurance measure to balance the influence of the interviewer during the qualitative data collection (Woodhouse, 2005). Data collected during the empirical study, the task analysis and the in-depth interviews were then analysed using an interview checklist and a recording marker system in the Morae software to determine the information-seeking behaviour of individual participants. Analysis of the data will provide an in-depth snapshot of how this particular group of Net Gen students are seeking information and using the Internet/Web. The data will also indicate any emerging trends in the information-seeking behaviour of young adult Internet users and how much their information-seeking behaviour is affected by their personal culture of use in the electronic 
environment. This paper will present the findings from the in-depth interview and the preliminary findings from the tasks which are still undergoing analysis.

\section{Findings}

A major finding from the anonymous Web survey is that there are no significant clusters in this group. The way participants are using technology, the types of technology they are using, where/how they acquired their skills in using the Internet and their levels of confidence, is extremely homogenous. As indicated in Figure 1, most of the participants fall within a standard deviation of the means for level of confidence (Affective Domain) and level of use (Effective Domain). There are small populations who fit into the low confidence/low use (LC/LU-NG) and high confidence/high use (HC/HU-NG) categories, but in terms of the whole group, these are not large enough to be significant. There are very small numbers who fall into the low confidence/high use (LC/HU-NG) and high confidence/low use (HC/LU$\mathrm{NG}$ ) categories. If this is an accurate finding, the homogenous nature of this survey group should also be evident in the follow-up interviews and task analysis. What students say they are doing anonymously should also correlate with what they say they're doing in a face-toface interview and with what they are actually doing in the tasks. If this particular survey group is typical of Net Generation users, the results should also reflect findings from other research studies.

Correlation analysis was also conducted using the Web survey data to establish any further relationships between how the participants were using technology, their levels of use and levels of confidence. This dataset supported earlier findings from the Web survey. This is an extremely homogenous group and participants are using technology according to their needs rather than ubiquitously. The interview results also followed this pattern, although there were some interesting additional findings. To enable a visual comparison between the students interviewed, the results were transferred to an Excel spreadsheet, with positive results appearing in yellow and negative results in blue. The homogenous nature of this group is very apparent in the bands of colour that appear in the spreadsheet results.

\section{Levels of use (Effective Domain)}

The Net Generation theorists were right about the frequency of use for members of this generation. Most of the interviewees reported using the Internet on a daily basis, even those who disliked using it. They are also very confident users.

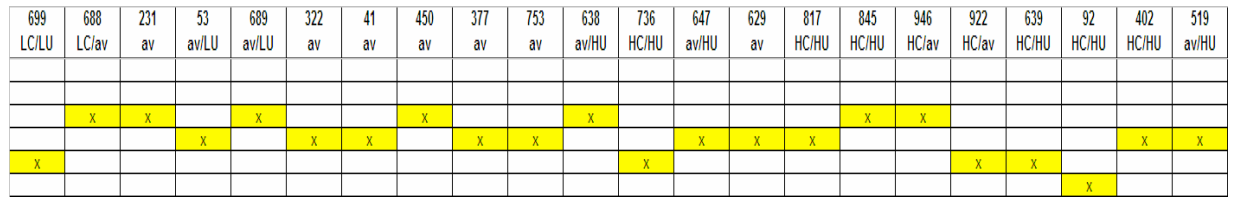

Figure 2: Interview males - personal rating of ability (confidence)

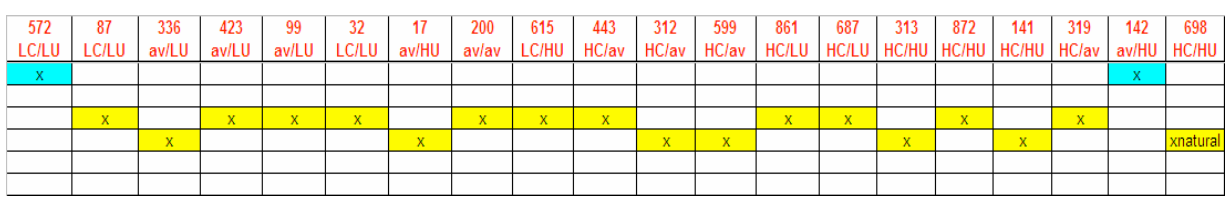

Figure 3: Interview females - personal rating of ability (confidence)

\section{Legend}

Rows: top - bottom

Beginner/non-user

Gaining confidence Average Good Very good Expert 


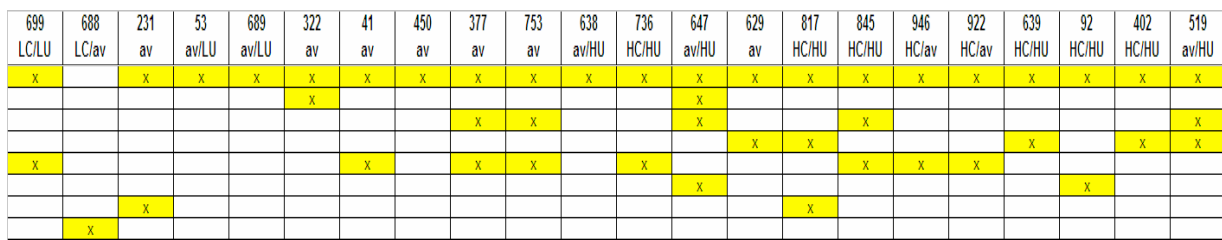

Figure 4: Interview males - frequency of use

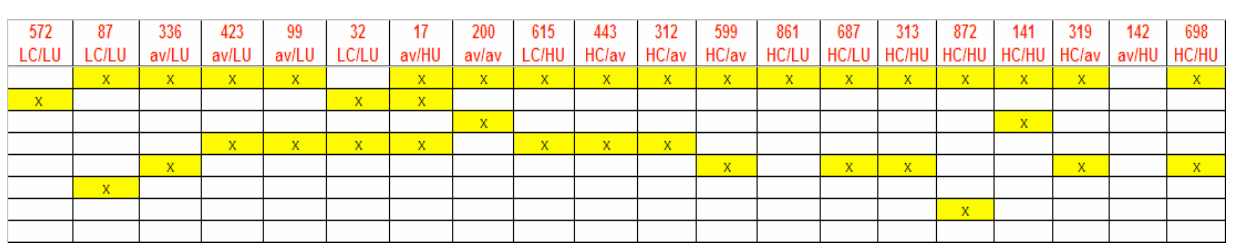

Legend

Rows: top - bottom

Daily

Daily/only at uni

At work

1-2 hrs/day average

Several hrs/day

Frequently

Computer on $24 / 7$

2-3 times/week, infrequently

Figure 5: Interview females - frequency of use

The interview results correlate closely with the Web survey results. This particular group of Net Geners are confident users of technology and most are using the Internet on a daily basis. Most of the survey group reported using the Internet often $(19 \%)$ or very frequently $(72 \%)$ during the previous 3 months. They also rate their abilities highly in comparison to other members of their peer group outside university and in comparison to the rest of their generation. A small number of interviewees believed the Net Generation myth and felt that the information-seeking behaviour of younger members would be better because they had had access to technology longer.

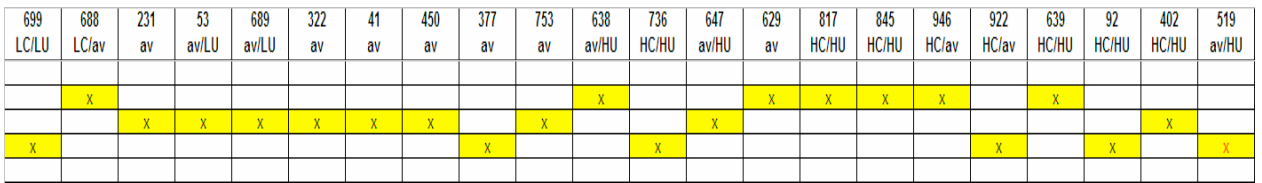

Figure 6: Interview males - information-seeking behaviour, personal rating

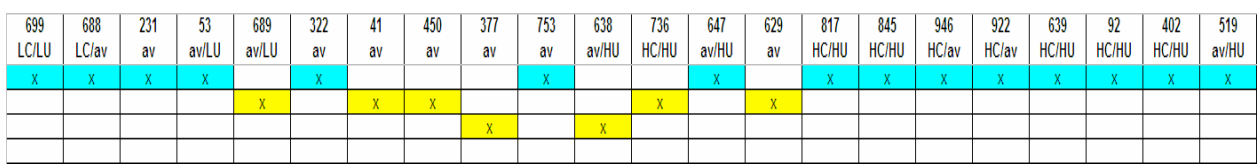

Figure 7: Interview males - information-seeking behaviour, compared to peers outside university

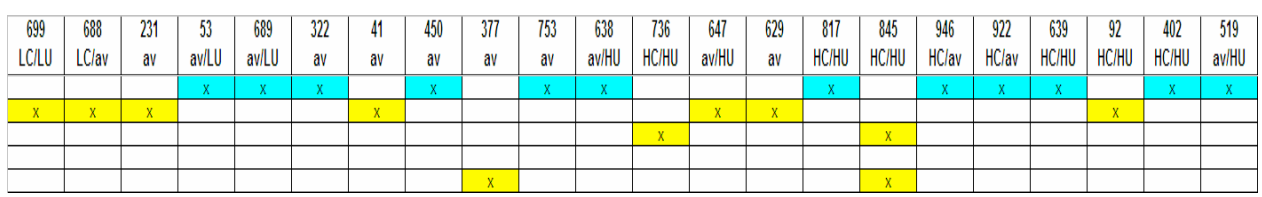

Figure 8: Interview males - information-seeking behaviour, compared to The Net Generation

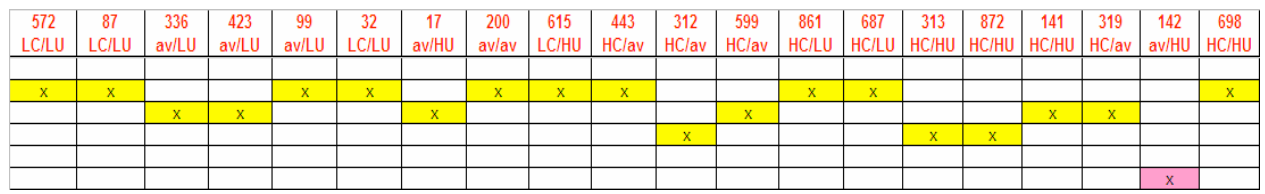

Figure 9: Interview females - information-seeking behaviour, personal rating

\section{Legend}

Rows: top - bottom

Non-user/Beginner

Average

Good

V Good

Expert

\section{Legend}

Rows: top - bottom

Less than me

Same as me

Better than me

Much better than me

\section{Legend}

Rows: top - bottom

Non-user/Beginner

Average

Good

V Good

Expert

Audio failed 


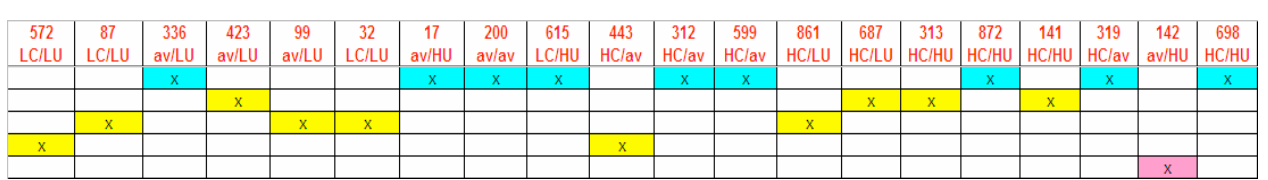

Figure 10: Interview females - information-seeking behaviour, compared to peers outside university

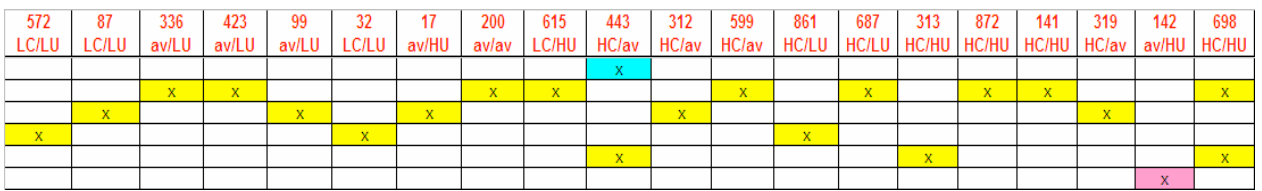

Figure 11: Interview females - information-seeking behaviour, compared to The Net Generation
Less than me Same as me Better than me Much better than me Audio failed

Less than me Same as me Better than me Much better than me Younger Net Gen much better than me Audio failed

Results from the correlation analysis indicate that frequency of use does have a positive impact on how young people feel about their ability to use the Internet successfully, particularly for finding information, communication and entertainment (see Appendix 1, Table 1: Correlation coefficients and levels of confidence). Is this a case of familiarity breeds contempt? As software developers create programs that are 'smart' and super user-friendly, where embedded artificial intelligence (AI) processes information in a pre-determined manner, does this lead young people into a false sense of confidence about their ability to find and use information? While $69.981 \%$ of the group reported that they felt confident about judging the reliability of the information they found on the Internet, few of the follow-up students interviewed planned their information-seeking when using search engines to search the Internet and a number admitted they had problems when trying to find information again.

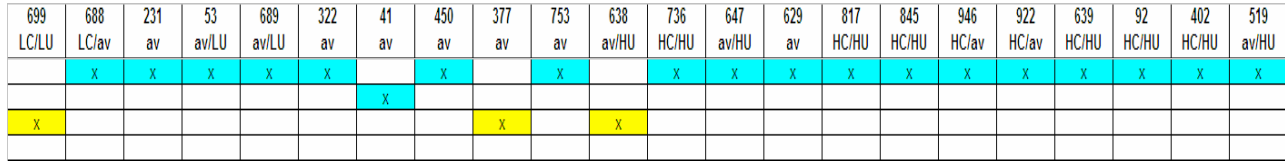

Figure 12: Interview males - information-seeking behaviour, planning

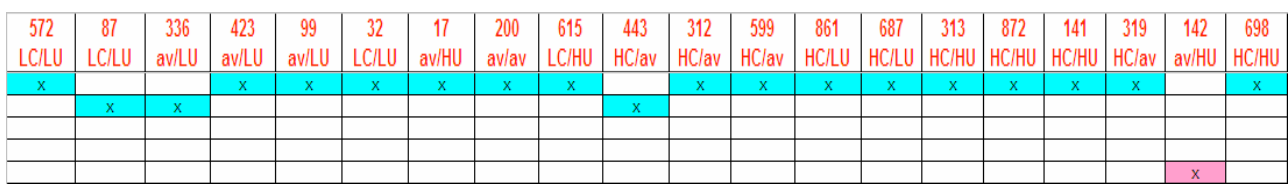

Figure 13: Interview females - information-seeking behaviour, planning

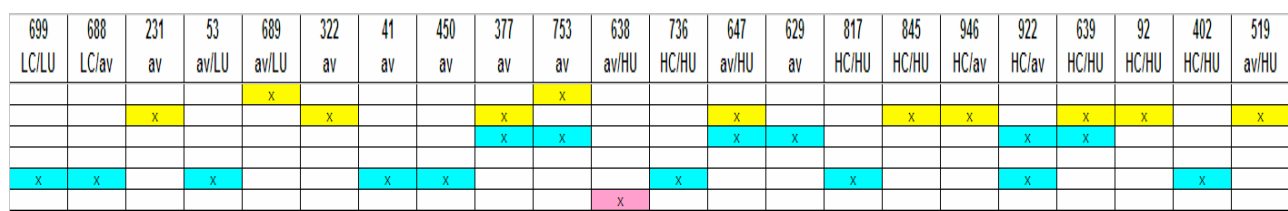

Figure 14: Interview males - re-finding information

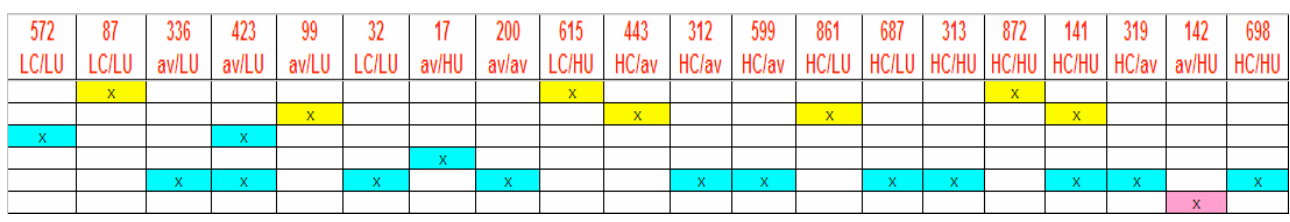

Figure 15: Interview females - re-finding information

\section{Legend}

Rows: top - bottom

No/not really

Sometimes

Usually

Always

Audio failed

Legend

Rows: top - bottom

Always

Usually

Yes - If I have saved information

Yes - personal use only

No/not always

Q omitted/audio failed/no

clear answer 
A small number of the student tasks have also been analysed and reveal that students spend a good deal of their time revisiting sites when searching for information. Each task lasted fifteen minutes. Figures 16 and 17 show the activity of a male student from the high confidence/high use (HC/HU-NG) category.

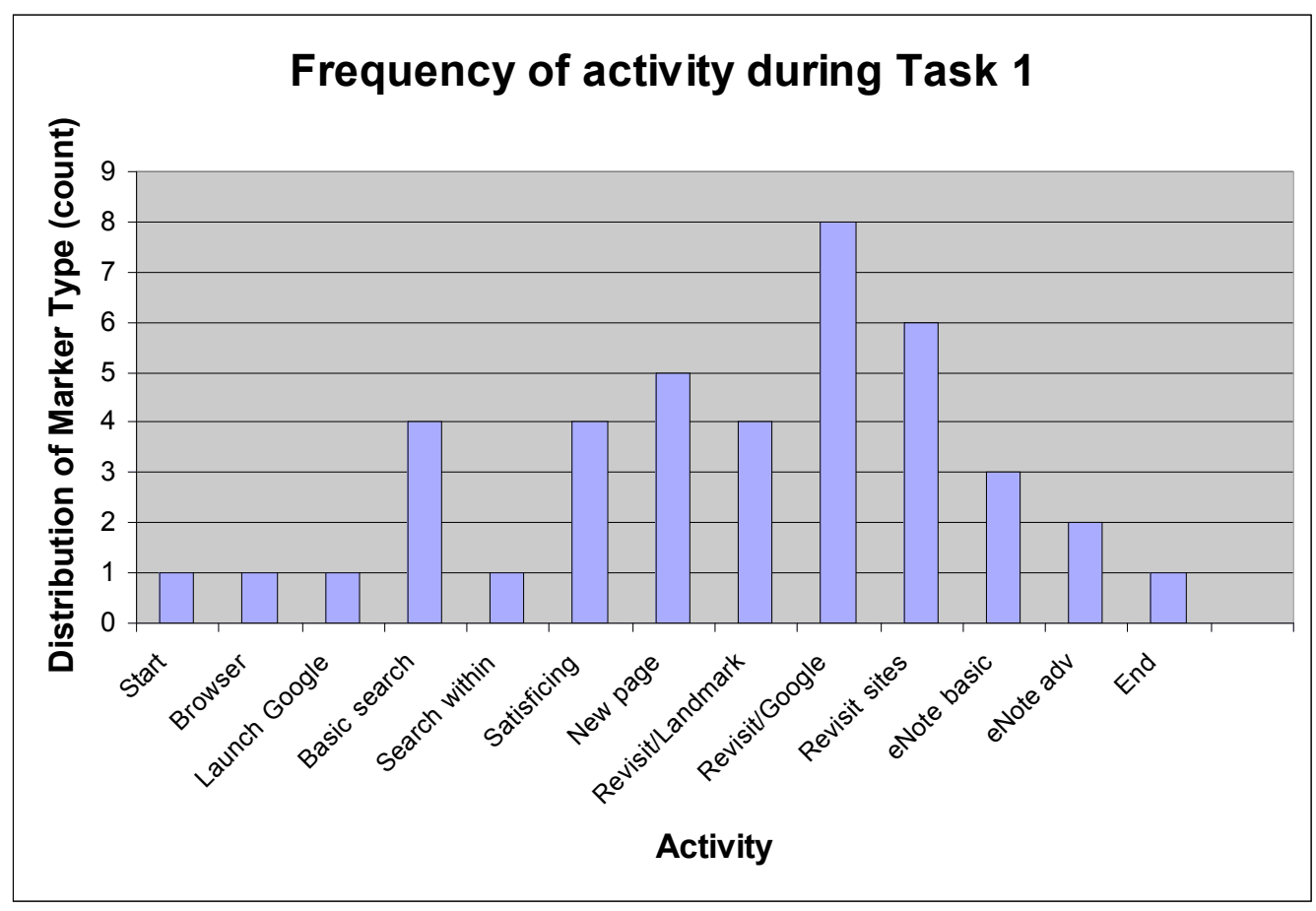

Figure 16: Student \#639, frequency of activity during task 1

Of the forty participants in the follow-up study only two students completed one of the tasks. In this case the student did not complete the first task. At no time did the participant appear to be frustrated with the task. Even though he had difficulty finding the information he required, he persisted with the task throughout. The student showed some evidence of multi tasking, but only if multi tasking is defined as moving between related tasks. When the participant found a site that contained some of the required information, he tended to stay with it and use it, rather than initiate a new search. He used the first site accessed from his original search as a landmark site and continually went back to this site as a starting point for his later searches. There was strong evidence of satisficing (accepting the first information available), he appeared to trust the search engine results and did not look further than the first two results in any of his searches. There was no evidence of planning and he did not revisit the task sheet.

The activity graph for task two where the student was looking for academic information is similar to task one, except he went to the library databases for his initial search. However, he did not use the library catalogue to find a book title and used EBSCO Host for the journal article even though this database was not particularly useful for the task. EBSCO is a journal database that is frequently used in secondary schools, an indication perhaps that his choice was based on prior familiarity. Searching in this database proved unsuccessful and he moved very quickly onto a new search in Wikipedia. Time elapsed in the library was less than three minutes. As in the first task, simple keywords were the main search method, multiple pages were open and he tended to revisit sites multiple times. While there was no planning evident, he did revisit the task sheet. Again, satisficing behaviour was evident, as he did not go beyond the first two results in the Google search and did not look 
deeper than the first four articles in the journal database, even when the title of the first result indicated that the article was not particularly relevant to the topic. While the student created electronic notes he cut and pasted ephemeral URLs (result of a search), which indicates a lack of understanding about searches and their results. He used Amazon to search for a book title. In both cases the student used the cursor to guide his reading of the text on the screen when reading in-depth, a phenomenon observed in other task analyses. This behaviour was also a major finding in the interview data, where students reported difficulties in reading and engaging with text on screen. There was a definite preference for printing and interrogating text on paper using traditional methods such as highlighting, a result that was also reflected in the Web survey where $85 \%$ of respondents felt the printer was either very important or essential.

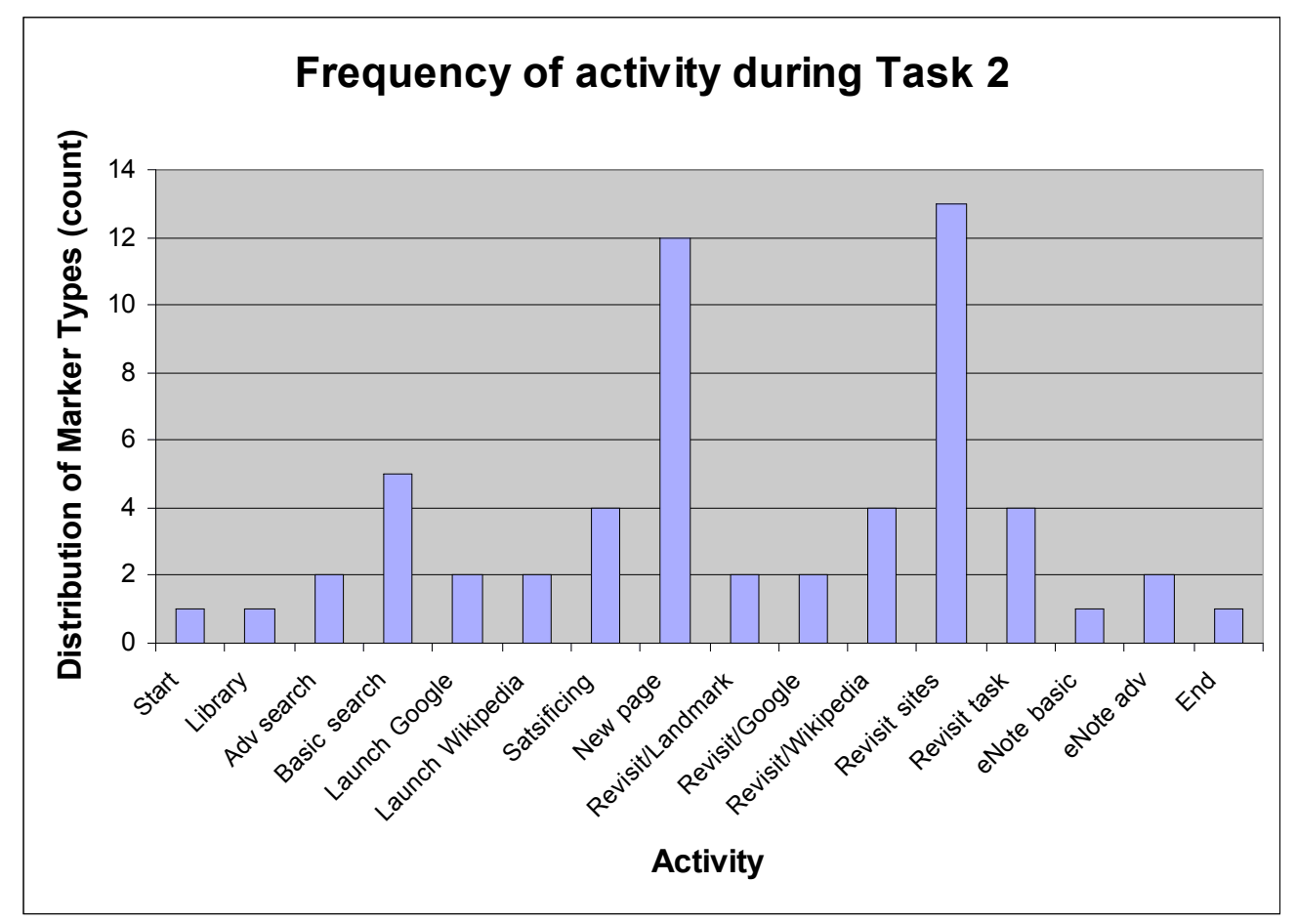

Figure 17: Student \#639, frequency of activity during task 2

In both tasks this $\mathrm{HC} / \mathrm{HU}-\mathrm{NG}$ student spent a large part of his searching time revisiting sites and switching between multiple open windows. He did not complete either task, did not appear to understand the limitations of the public domain Web, trusted the search engine results and information found in Wikipedia and often appeared to be lost in virtual space. The propensity of users in this age group to exhibit satisficing behaviour has been widely reported in the literature (Case, 2002; Nicholas et al., 2003; Fallows, 2005; Scott, \& O'Sullivan, 2005). As evidenced here, other studies have found that students use unsophisticated search strategies, tend to browse or use commercial search engines like Google and demonstrate a serious lack of understanding of the limits of the free Web (Griffiths, 2003; Everhart, \& Valenza, 2004; Sandvig \& Baiwa, 2004; Scott, \& O’Sullivan, 2005). Results from the in-depth interviews also indicated an unsophisticated culture of use where students use simple keywords to search for information. They use this method even if they have had some prior instruction in how to search the databases using Boolean search strategies. They also use the same method when searching within websites and in one case when searching in the journal titles section of the library. This culture of use is predicated on experiential learning and the results of the interviews and tasks analysed so far, indicate that 
it is extremely difficult to change. This characteristic has also been reported in the literature from the Illinois Mathematics and Science Academy (IMSA) 21st Century Information Fluency Program (2lCIF) research project. This project used pre-testing and post-testing after an intervention skills program in electronic search techniques, and found that many students reverted back to the culture of use they employed prior to the intervention (Barr et al., 2006). Such behaviour suggests that students come to serious information seeking in the electronic environment with an established culture of use that requires closer scrutiny by researchers and schools if we are going to change information seeking behaviour in the electronic environment.

Students in the interviews indicated that they are using Google almost exclusively when searching the Internet. They rarely go beyond the first results page and always use simple keyword searching. The way the Google algorithms work (most popular site visited first) coupled with this generation's tendency to satisfice when information seeking and their trust in the search engine results, means they are often unsuccessful or don't find information that is specific to their query. Students in the interviews also indicated that if the information they are seeking isn't recognisable in the first page of search results, then they will change their search terms/keywords. Many students used the term relevant when talking about search engine results, ie. if the search engine result appears to be relevant to my search then it must be the best information for me. Coupled with their trust in search engine results and confidence, this leads to an assumption of authority, ie. if the search engine result is relevant to my query then it must be authoritative or good information. This idea of relevance $=$ authority may also explain why most participants felt that they find what they are looking for most of the time. Many participants indicated that problems only arise when they are searching for very specific information. This trust in search engine results and participants' confidence also means that if they can't find anything after trying several searches, they will often assume that the information isn't available, rather than concluding that they can't find it. The reliance on keyword search methods across all areas (Google/search engines), closed systems/repositories (databases) and on the Web (Wikipedia) indicates a lack of sophistication in search methodology that will limit the quality of information retrieved.

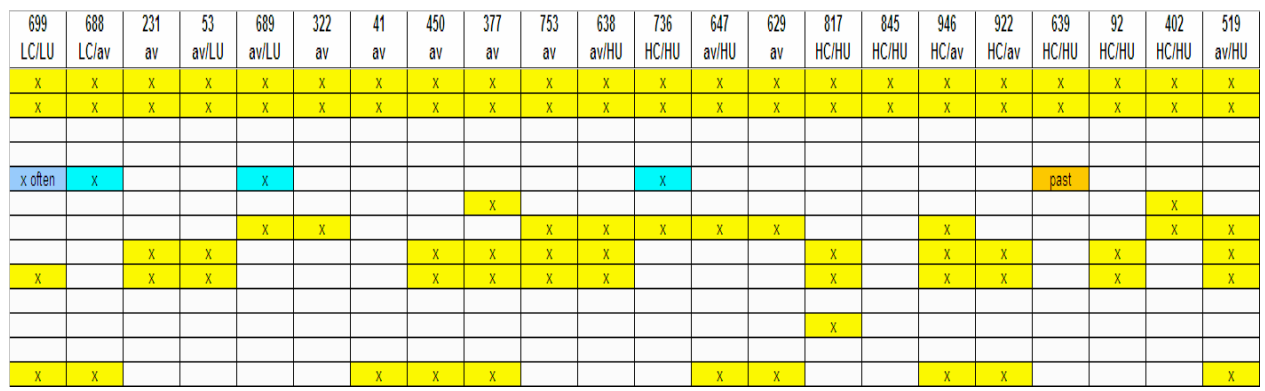

Figure 18: Interview males - information-seeking behaviour, Google

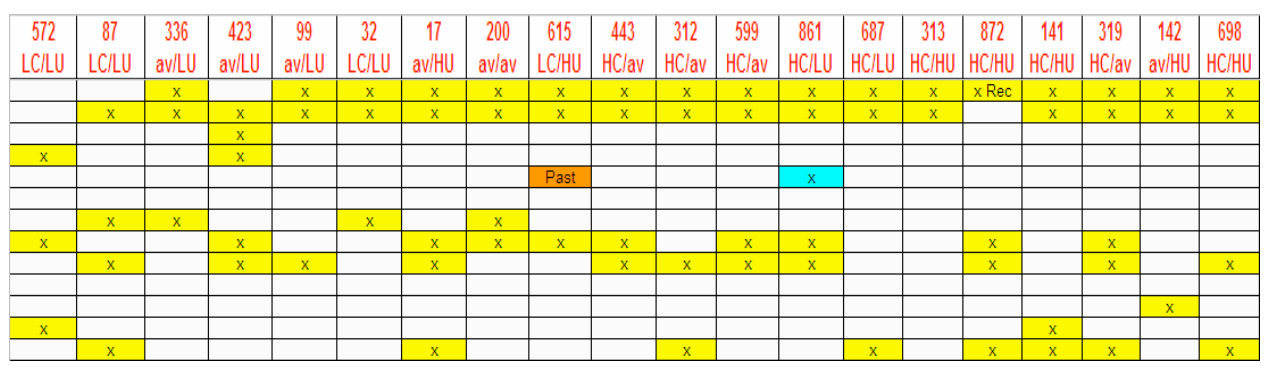

Figure 19: Interview females - information-seeking behaviour, Google

\section{Legend}

Rows: top - bottom

Culture of use - Searching

Google always use/usually

Google - use keywords

Adv search - sometimes

Sometimes, Google

Sometimes, other search engines Wikipedia use as landmark site Wikipedia use keywords Use Library/Web databases Use keywords - library databases Use Boolean search methods Use a sentence/phrase to search Not allowed to use public websites Relevance $=$ authority 
Students interviewed indicated that they trust the search engine results and assume that search engines such as Google list results with the most appropriate or relevant site for their search enquiry, first. One student commented that 'Google knows everything' and 'Google is king'. This anthropomorphisation of a search engine is an extreme example of this trust, where the student appears to be attributing intelligence to the search engine. This level of trust and confidence in search engines and the tendency to equate relevance with authority and accuracy, indicates that the young people in this group do not really understand how different search engines work. Undoubtedly, Google's success has been largely due to the search engine's uncluttered design (student comment), ease of use and the fact that any search will ensure the retrieval of some information. User-friendly access to information, where the technology appears to be able to do the work for the user is attractive to this generation of users (Everhart, \& Valenza, 2004) who are used to having fast access to other people, information and entertainment. Perhaps this explains the overt satisficing behaviour of this generation of users.

Some of the participants also felt that constant exposure to a lot of information enabled them to differentiate good information from 'rubbish'. However, the evaluative processes used by participants appears to be cursory or based on superficial methods such as looking to see if a web site has an education or government designator in the first part of the web site address (URL). The assumption here is that if the information is present on a web site with a government or education designator in the web address, then this makes it good information, even if there is a tilda $(\sim)$ in the URL which indicates that the site may not be part of the official site, but rather, a private individual who is using the organisation's server. Some students indicated that they will sometimes try to verify authorship or quality by looking for other websites that say the same thing, ie. the assumption that if information is common, then it is authoritative. This method is also flawed due to the nature of the Web, which promotes fluidity and facilitates the easy transfer and manipulation of information by multiple users. User-friendly access and the ability to manipulate information quickly and easily as part of everyday use, means that information shared via the Web tends to lose its original integrity as it is picked up and re-used by multiple users and disseminated around the global network. "There is no audit trail on the Internet. Information is subtly transformed and like Chinese whispers, the end product may bear no resemblance to the original in form, context or purpose" (Combes, 2004, ACEC). Thus, if information that is not authoritative and has little quality is circulated by enough people, it can eventually come to be regarded as true, good and even new, information. Hence, the very nature of the Web and how information is presented and circulated, can lead to a situation of compound ignorance, where poor quality information is constantly verified simply because it is being picked up and used by multiple users. This situation is exacerbated by search engines such as Google which present the most popular sites first as a search result, rather than the most appropriate or best information available for the query. Trust in search engine results, ease-of-use and the expectation that the technology will do the thinking for the user, are features of this particular group's information-seeking behaviour.

The majority of the students interviewed also admitted to using Wikipedia regularly, even though they were all aware that this is not considered a reliable academic source. Students were using Wikipedia as a landmark site, as a general reference site for definitions of terms and to acquire links to other information on the Web. While they weren't citing Wikipedia in their assignments (because they had been warned not to), they were still using it, sometimes as their only information source. The second task (academic) required students to have an understanding of the term lifelong learning, which is defined in Wikipedia as adult 
learning. Since none of the interviewees planned their information task and Wikipedia was the most frequently and only source used, the information retrieved for the task was not accurate.

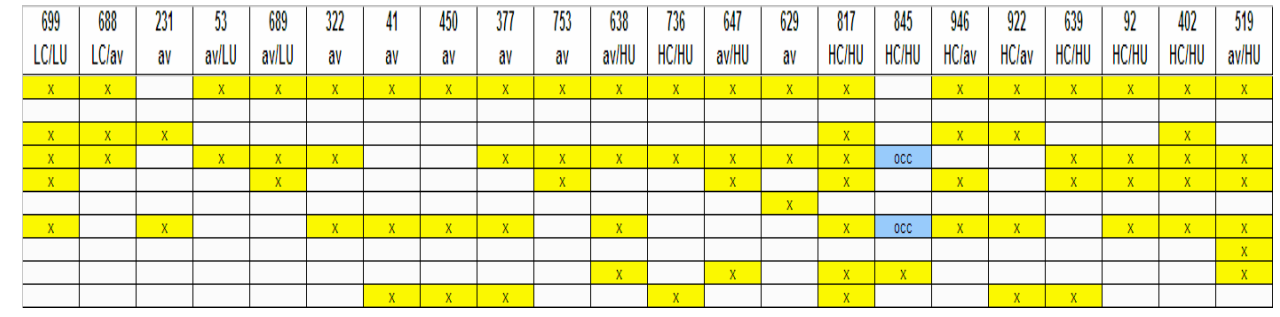

Figure 19: Interview males - information-seeking behaviour, Wikipedia use

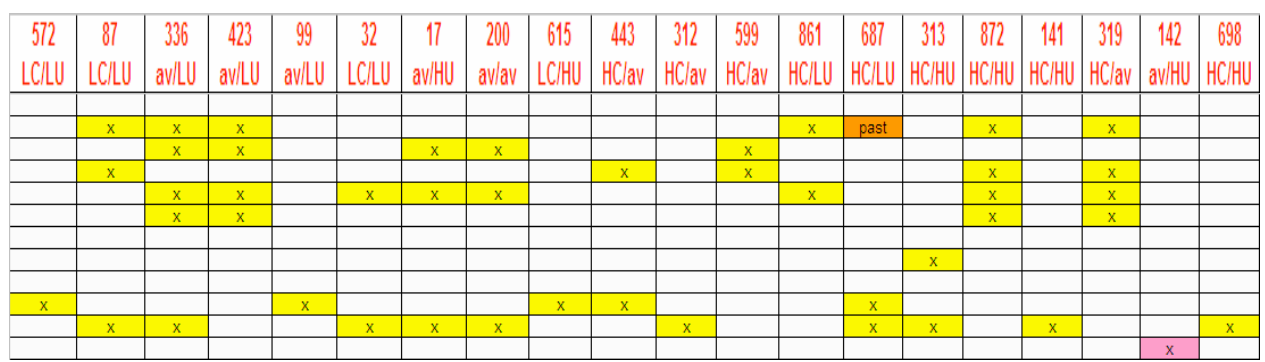

Legend

Rows: top - bottom

\section{Wikipedia Use}

Yes

Sometimes

Don't cite it

Starting point/general reference Find and use links to information

For academic use, reliable information

Yes - for personal use Wikipedia editor

No/Not allowed to use for uni Unreliable for uni

Audio failed

Figure 19: Interview females - information-seeking behaviour, Wikipedia use

All participants are using keyword searching techniques exclusively. Participants' consistently responded when questioned about their method that 'it works for me', 'guess it's habit' and 'if it ain't broke why fix it?' These responses indicate that the participants in this group have a defined culture of use when seeking information on the Web using search engines. They also transfer this culture of use when searching for information elsewhere, even after receiving specific instruction on how to search for information using other techniques. Hence, they are also using keyword searches in the closed system of the university databases (if they are using them), and Wikipedia.

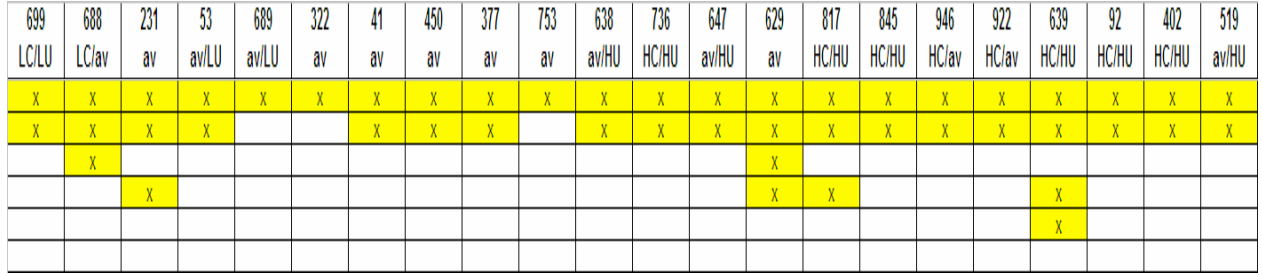

Figure 19: Interview females - information-seeking behaviour, culture of use

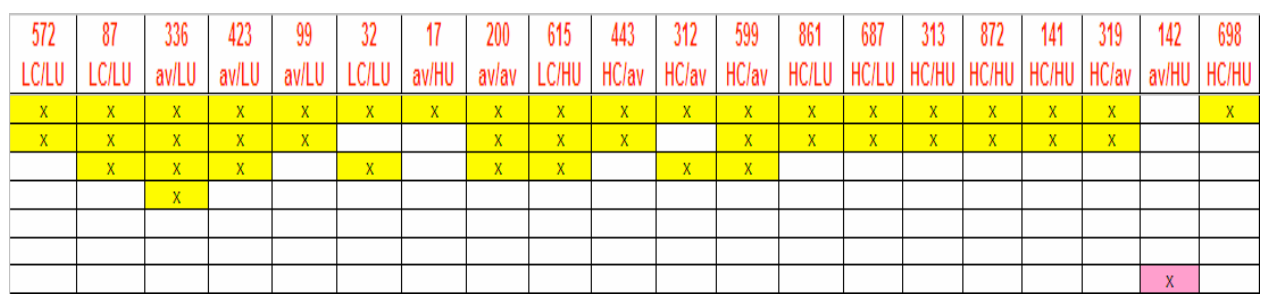

Figure 19: Interview females - information-seeking behaviour, culture of use

\section{Legend}

Rows: top - bottom

Use same method

Yes, always

Reasons

Works for me - habit Easy - one method Reliable, esp Google Google - simple, uncluttered interface

No

Audio failed

Another interesting result from the interviews is how these members of the Net Generation are using social networking sites. Students are using social networking sites such as MySpace and FaceBook as an alternative to email (some even described their use in these terms). They rarely diarise and publish about themselves in any depth (the small number of 
students who do, admitted to exaggerating their profile information for fun). Rather, they use the technology to keep in contact with established or previous friendship groups (friends from primary school with whom they have lost contact). Only one student used it regularly to contact outsiders and this student admitted to having some bad experiences (illegal activities) when younger (under-age) when he 'got in with the wrong crowd'. Participants are using the social networking sites as an alternative to email because it provides a centralised space that is more protected from spam email and as an alternative to chat $/ \mathrm{msn}$ because they have more control over their time. When communicating in MySpace or FaceBook there is not the expectation of an immediate reply. Several respondents indicated that friends did not get offended about a lack of immediate response when using the social networking sites for communication as much as when using real time communication such as msn or chat or even email. Fifty percent of the interviewees are not using and have never used these sites. A number of respondents indicated that they used to use the social networking sites, but have now moved on and don't use them any more due to different time commitments, work and study. One student stopped using them because she found the sites became a forum for animosity between different friends/ship groups, a situation over which she had limited or no control. Most students also limit the access on their social networking sites and only communicate with people they know or have known in the past. They are not politically active nor are they socially inclusive when out on the Web.

This adaptation of technology is an indication that this generation are discerning users. They will adapt the technology to suit their needs and move on when it is no longer useful or their needs change. As teachers we are continually being exhorted to get into their (students') space, as everyone is social networking. This type of reasoning is corporate driven and if the results from this study are an accurate snapshot of how this generation are using social networking sites, then perhaps we need to rethink why we would want to use them in the classroom.

\section{Conclusion}

Confidence is perhaps the key to understanding how this generation use technology, an aspect the Net Generation theorists observed and postulated on at such length. They misinterpreted confidence and assumed that this also translated into intentional, meaningful and effective information-seeking. The students are confident which means they are using the Internet on a daily basis, even those who are less confident. This confidence may be what the Net Generation theorists got right and is borne out of familiarity with the technology. The technology is a ubiquitous part of their information landscape and something they have never been without. Even if they dislike using technology, they still use it and with confidence. This confidence also means that they will try new technologies. This attitude towards technology also means they use it in a discerning manner, and pick and choose technologies that suit their needs at a particular time. Hence, participants report that their usage patterns have changed, as their lifestyle and information needs have changed. This explains why the levels of use in the Web survey were quite low, as the metric was based on the Net Generation theory which postulates that the Net Generation uses a wide range of technologies. This is not the case. They use technology to be connected more than anything else, and they use it for entertainment. They use it for finding information when the need arises and they have acquired a culture of use when seeking information via electronic means.

While the technology is playing a part in the development of this culture of use, it is also being driven by the fact that students are being left to learn their information-seeking skills on their own by experimentation. This lack of formal information-seeking skills 
instruction is due to the fact that educational administrators and teachers believe the myth promulgated by the Net Generation theorists and assume that students (Generation Y = digital natives) already have the skills to locate information using electronic resources, and to interrogate and consequentially use this information to meet their needs. The assumption that students have the skills to locate information in the virtual environment simply because they are familiar with technology and confident about using it, has meant that information-seeking behaviour amongst members of the Net Generation is unsophisticated, demonstrates a culture of use that is hard to change and the result of a lack of formal information literacy education. They have poor Internet literacy skills, rely on keyword searching, trust search engine results and as a consequence, exhibit a high level of satisficing. This generation's lack of understanding of how the Web works coupled with high levels of confidence, means they often fail to realise they don't know and assume that if they can't find it on the Web then it doesn't exist. If schools don't take steps to teach this generation of students how to use electronic sources effectively, then our future citizens will be unable to operate in a world where information is the key to educational, social and economic success. The world and technology will continue to move forward and the information landscape will become more complicated, overloaded and dense, as business and government place everything including service delivery online. The Net Generation and those who follow, however, will remain lost forever in virtual space.

\section{References}

Banwell, L. and Gannon-Leary, P. (2000). JUBILEE: Monitoring user information behaviour in the electronic age. OCLC Systems and Services 16 (4).

Barr, D., Garrett, P., Balzer, D., Heine, C. \& Bob Houston (2006). Search challenges as assessment tools: A collaboration between the library and the $21^{\text {st }}$ Century Information Fluency Project in Illinois. Proceedings of the ALIA Click06 Biennial Conference. Retrieved May 15, 2008 from http://conferences.alia.org.au/alia2006/Papers/David Barr_and Paula_Garrett.Pdf

Case, D.O. (2002). Looking for information: A survey of research on information seeking, needs and behavior, California: Academic Press.

Combes B. (2004). The culture of information usage, plagiarism and the emerging Net Generation. paper presented and published in Research..., Reform..., Realise the potential! proceedings of the Australian Computers in Education Conference (ACEC) 2004, July, Adelaide, South Australia.

Combes, B. (2006). Techno Savvy and All-knowing or techno-oriented? Informationseeking Behaviour and the Net Generation. IASL Reports 2006: The Mutiple Faces of Literacy, Reading, Knowing, Doing, Lisbon, Portugal.

Combes, B (2007a). The search for information and the Net Generation. Cyberspace, Dworld, E-learning: Giving libraries and schools the cutting edge, The 2007 IASL Conference, National Taiwan Normal University, Taipei, Taiwan, 16-20 July 2007

Combes, B. (2007b). Techno savvy or just techno oriented? How do the 'Net Generation' search for information? ASLAXX Biennial Conference: Hearts on fire, sharing the passion, Adelaide, Australia, 2-5 October 2007.

Dorman, S.M. (2000). Implications of growing up digital. Review of Tapscott's Growing up digital. The Journal of School Health, 70(10) 420-422

Educational Testing Service (ETS). (2006). 2006 ICT literacy assessment: Preliminary findings. Retrieved May 15, 2008 from www.ets.org/ictliteracy 
Fallows, D. (2005). Search engine users, PEW Internet \& American Life Project. Retrieved May 15, 2008 from www.pewinternet.org/

Griffiths, J.R. and Brophy, P. (2002). Student searching behaviour in the JISC Information Environment. Ariadne, No.33. Retrieved May 15, 2008 from http://www.ariadne.ac.uk/issue33/edner/

Livingstone, S., Bober, M. and Helsper, E. (2005). Internet literacy among children and young people: Findings from the UK Children go online project. London School of Economics. Retrieved May 3, 2007 from www.children-go-online.net

Nicholas, D., Dobrowolski, T., Russell, C. \& Withey, R. (2003). Digital information consumers, players and purchasers: Information seeking behaviour in the new digital interactive environment. Aslib Proceedings, 55 (1/2) 23-32

Nicholas, D., Rowlands, I. and Huntington, P. (2008). The information behaviour of the researcher of the future: A CIBER briefing paper. University College London. Retrieved May 14, 2008 from http://www.jisc.ac.uk/whatwedo/programmes/resourcediscovery/googlegen.aspx

Oblinger, D. \& Oblinger, J. (2005). Is it age or IT: First steps towards understanding the Net Generation in Oblinger, D. \& Oblinger, J (Eds), Educating the Net Generation. Retrieved May 15, 2008 from http://www.educause.edu/educatingthenetgen

Scott, T.J. \& O'Sullivan, M.K. (2005). Analyzing student search strategies: making a case for integrating information literacy skills into the curriculum. Teacher Librarian, 33 (1) 21-25.

Skiba, D. (2003). The Net Generation: Implications for nursing education and practice. In NLN Living Book. Retrieved May 25, 2008 from http://electronicvision.com/nln/chapter01/

Tapscott, D. (1998). Growing up digital: The rise of the Net Generation, McGraw-Hill, New York.

Woodhouse, M. (2005). Using in-depth interviews to evaluate deep learning in students who use online curriculum: a literature review. TILC: Information, Libraries and eLearning, p. 214-225.

\section{Biographical notes}

Barbara lectures for the School of Computing and Information Science at Edith Cowan University, Western Australia. Her major research interest areas include the role of teacher librarians in education, plagiarism, online learning and distance education particularly for first time users, information literacy, policy and planning. Barbara's $\mathrm{PhD}$ research is examining the informationseeking behaviour of the Net Generation. She has published in a number of journals, presented at conferences worldwide and is the Vice President Advocacy and Promotion for the IASL.

\section{Statement of Originality}

This statement certifies that the paper above is based upon original research undertaken by the author and that the paper was conceived and written by the author(s) alone and has not been published elsewhere. All information and ideas from others is referenced. 


\section{Appendix 1}

\begin{tabular}{|l|l|c|}
\hline \multicolumn{2}{|l|}{ Correlation Matrix: Net Generation } \\
\hline Confidence (Affective Domain) & Q5 Self-evaluation/rating \\
\hline Q1 & Use for study prior to university & $\mathrm{p}<0.0005, \mathrm{r}=0.458685$ \\
\hline Q2 & Use of online study materials prior to university & $\mathrm{p}<0.0005, \mathrm{r}=0.463205$ \\
\hline Q3 & Personal use for study prior to university & $\mathrm{p}<0.0005, \mathrm{r}=0.492579$ \\
\hline Q8 & Level of personal use & $\mathrm{p}<0.005, \mathrm{r}=0.277726$ \\
\hline Q9 & Length of time using the Internet & $\mathrm{p}<0.005, \mathrm{r}=0.291835$ \\
\hline Q10iv & Buying and selling & $\mathrm{p}<0.005, \mathrm{r}=0.278185$ \\
\hline Q14i & Use for study & $\mathrm{p}<0.005, \mathrm{r}=0.26003$ \\
\hline Q14ii & Communication - family/friends & $\mathrm{p}<0.005, \mathrm{r}=0.275997$ \\
\hline Q14v & Planning and organisation & $\mathrm{p}<0.005, \mathrm{r}=0.282334$ \\
\hline Q14vi & Entertainment & $\mathrm{p}<0.005, \mathrm{r}=0.287442$ \\
\hline Q15i & Ability to find information & $\mathrm{p}<0.0005, \mathrm{r}=0.33586$ \\
\hline Q15iv & Ability to organise information & $\mathrm{p}<0.005, \mathrm{r}=0.289029$ \\
\hline Q15v & Ability to store information & $\mathrm{p}<0.005, \mathrm{r}=0.27841$ \\
\hline Q15vi & Ability to find information again & $\mathrm{p}<0.0005, \mathrm{r}=0.321041$ \\
\hline Q16vi & Like using for entertainment & $\mathrm{p}<0.005, \mathrm{r}=0.271187$ \\
\hline
\end{tabular}

Table 1: Correlation coefficients and level of confidence

\section{Correlation Matrix: Net Generation}

\begin{tabular}{|l|c|}
\hline Levels of use (Effective Domain) & Q8 Use/personal \\
\hline Q10iv Buying and selling & $\mathrm{p}<0.005, \mathrm{r}=0.268393$ \\
\hline Q10v Planning and organising & $\mathrm{p}<0.005, \mathrm{r}=0.270928$ \\
\hline Q10vi Entertainment & $\mathrm{p}<0.0005, \mathrm{r}=0.54005$ \\
\hline Q11iv Communication - msn or chat & $\mathrm{p}<0.0005, \mathrm{r}=0.482838$ \\
\hline Q11ix Peer-to-peer file sharing & $\mathrm{p}<0.005, \mathrm{r}=0.392496$ \\
\hline Q11xi Web blogs & $\mathrm{p}<0.0005, \mathrm{r}=0.299964$ \\
\hline Q17 Gender - male & $\mathrm{p}<0.001, \mathrm{r}=0.271107$ \\
\hline
\end{tabular}

Table 2: Correlation coefficients and levels of use

\begin{tabular}{|l|c|}
\hline Correlation Matrix: Net Generation - \\
\hline Gender & Q17 Gender (males) \\
\hline Q8 Personal use per week & $\mathrm{p}<0.005, \mathrm{r}=0.271107$ \\
\hline Q10iv Buying and selling on the Internet & $\mathrm{p}<0.001, \mathrm{r}=0.30845$ \\
\hline Q11ix Use peer-to-peer file sharing & $\mathrm{p}<0.005, \mathrm{r}=0.327595$ \\
\hline $\mathrm{Q} 13 x$ ii Books/magazines for skills & $\mathrm{p}<0.005, \mathrm{r}=0.275877$ \\
\hline
\end{tabular}

Table 3: Correlation coefficients and gender (male) 